\title{
Holistic Movement for Diabetes
}

\author{
Rammal J* \\ Founder, JSport, Lebanon
}

*Correspondence: Julie Rammal, Founder, JSport, Lebanon

Received on 22 April 2020; Accepted on 05 May 2020; Published on 14 May 2020

Copyright $\odot 2020$ Rammal J. This is an open access article and is distributed under the Creative Commons Attribution License, which permits unrestricted use, distribution, and reproduction in any medium, provided the original work is properly cited.

\section{Introduction}

The human body is made to move in a language that the body understands, reacts to, and improves vitality, flexibility, natural strength, focus and discipline. If it does not move enough or correctly, it can develop energy blocks, stagnation, sickness and more.

\section{Holistic Movement}

The holistic movement focuses on sharing movement with a language that the body recognizes and reacts to. The holistic methodology focuses on connecting the human body, mind, and soul naturally back into the system to find health, happiness, energy and youthfulness. It is the movement designed for present and the future. It effectively unblocks the body, renews its energy, reconnects it back to itself and a higher source of power.

Physical activity is very important for adults who have type 2 diabetes for overall well-being and blood sugar. The body must move naturally through natural movement such as brisk walking, hiking, jogging, swimming, stretching, and holistically tone and strengthen the system. Lack of proper movement or movement can dramatically affect the health and energy of the body, mind, and soul.

In our current and future era, movement may become a challenge as our lives will become more sedentary, stressed, connected to technology, and time may become less available. The holistic movement offers an intelligent way to train, detox, and strengthen the body naturally as it offers a mix of integrated ancient therapies, movements, and holistic stretching to revitalize and boost energy and health. The movements have been well designed to bring balance and equilibrium into all three systems naturally.

For the majority, the current human species is disconnected and unaligned to the universal field of energy and themselves. As a result, this lack of connection has weakened the human species on all levels. The future human species must equally inter-connect within, above, and to technology to surpass the challenges that are poised on the creation and existence of the human species. If not, the human species will transform into a trans robotic species and will have little or no emotions, lack of thought, motivation, decreased health, and will solely perform tasks as their body and mind systems have been programmed to do so. These changes are already visible in the movement, health, living, and medical sectors. In addition, diabetics are at higher risk with the evolutionary changes in our environment, lifestyle, and movement. 


\section{Conclusion}

In conclusion, the holistic movement is the movement of the future to help people inter-connect to themselves, train body and mind, boost healing, and to live a naturally healthy life. The movements have been intelligently designed to speak and understand the body and receive effective body and mind results. 